\title{
Switching the Magnetic Vortex Core in a Single
}

\section{Nanoparticle}

Elena Pinilla-Cienfuegos, ${ }^{\dagger}$ Samuel Mañas-Valero, Alicia Forment-Aliaga* and Eugenio

Coronado*

Instituto de Ciencia Molecular (ICMol). Universitat de València, Catedrático José Beltrán 2, E46980 Paterna, Spain.

\section{Corresponding Author}

* Prof. Eugenio Coronado. E-mail: eugenio.coronado@uv.es

* Dr. Alicia Forment-Aliaga. E-mail: alicia.forment@uv.es

\section{S.I. 1 Tip-Calibration and Tip influence}

The coercive field of the commercial MFM probes (MFMR-Nanosensors tips) was calibrated by using the procedure proposed by M. Jaafar et al. ${ }^{1}$ The magnetic measurements were performed at almost the same low temperature, $4.5 \mathrm{~K}$, used in the MNPs experiments and at the same tip-sample distance, $Z_{\text {lift }}=100 \mathrm{~nm}$. Using a commercial hard disk piece as reference sample, we measured the changes of the magnetic images contrast on the hard disk bits when sweeping the out-of-plane magnetic field applied $\left(B_{e x t}\right)$. Due to the broad coercive field of

\footnotetext{
${ }^{1}$ Jaafar, M.; Asenjo, A.; Vázquez, M. Calibration of Coercive and Stray Fields of Commercial Magnetic Force Microscope Probes. IEEE Trans. Nanotechnol. 2008, 7, 245-250.
} 
the hard disk, the changes in the magnetic contrast gave us information about the magnetization of the MFM tip, since in this case the magnetization of the tip was reoriented with the change of $B_{\text {ext }}$, while the magnetization of the hard disk remained unmodified. From the analysis of the evolution of the MFM contrast versus magnetic field, we deduced the coercivity of the MFM probe. The value obtained for the out-of-plane measurements at 4.5 $\mathrm{K}$ was $\mathrm{H}_{\mathrm{c}} \sim \pm 750 \mathrm{Oe}^{2}$

The total magnetic field, $\vec{B}$ that is applied to the MNPs while the tip scans the sample is the resultant of the magnetic field produced by the tip plus the external field applied, $\overrightarrow{\mathrm{B}}=\overrightarrow{\mathrm{B}}_{\text {tip }} \pm \overrightarrow{\mathrm{B}}_{\text {ext }}$. When $\mathrm{B}_{\text {ext }}$ is applied in the same orientation than the magnetization of the tip, $\mathrm{B}_{\text {tip }}$, both fields are added giving rise to a total field higher than $\mathrm{B}_{\mathrm{ext}}$. However, when $\mathrm{B}_{\mathrm{ext}}$ points to the opposite orientation, there will be a decrease in the resulting field acting over the MNP (Figure S1).

${ }^{2}$ Pinilla-Cienfuegos, E. et al. Imaging the Magnetic Reversal of Isolated and Organized Molecular-Based Nanoparticles Using Magnetic Force Microscopy. Part. Part. Syst. Charact. 2015, 32, 693-700. 

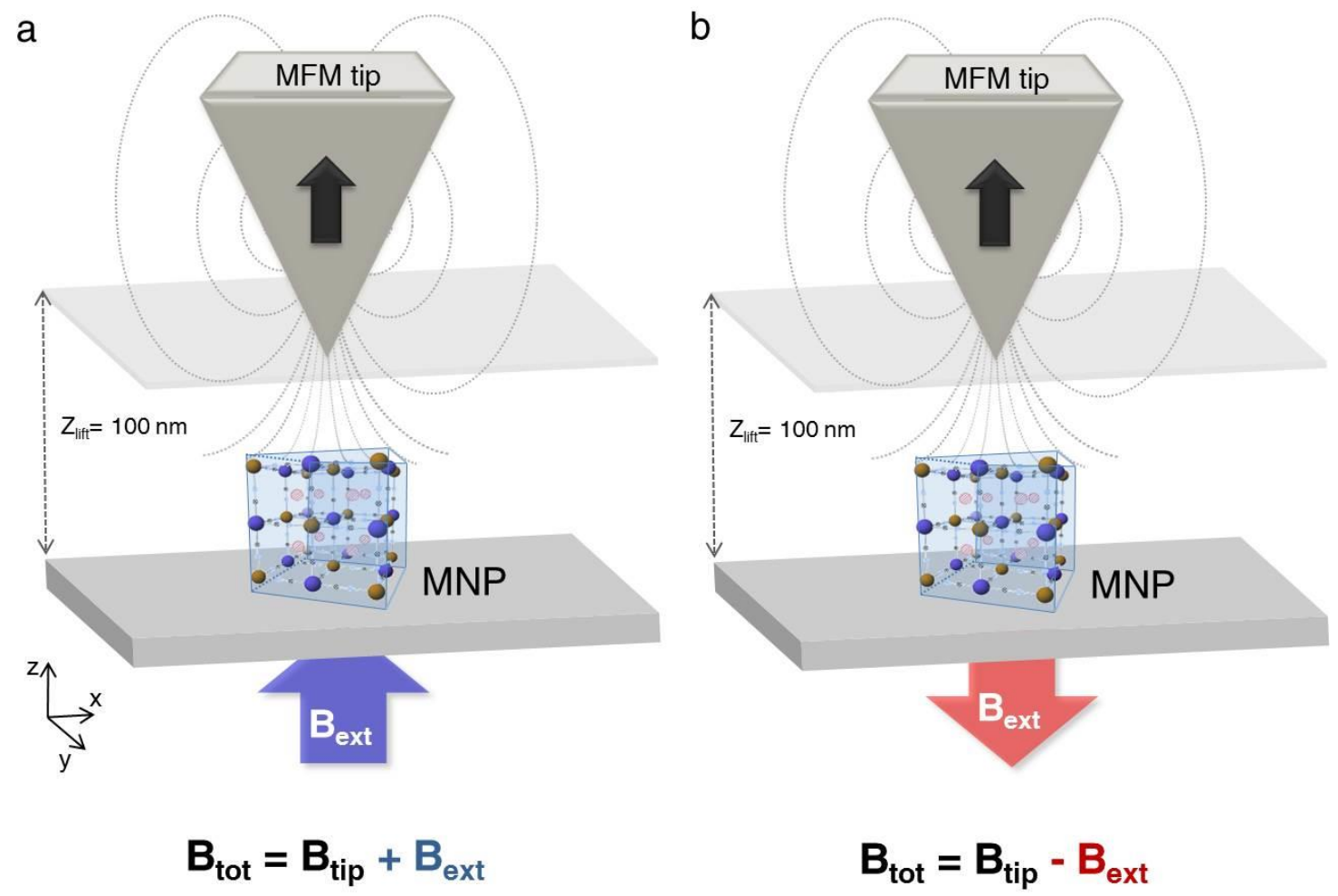

Figure S1. Scheme of the tip-sample relative position and the orientation of the external fields that act over the magnetic nanoparticle. (a) When the orientation of both fields, external and the stray of the tip, are in the same orientation, both are added; (b) When they have opposite orientations, they can compensate each other partially or totally.

\section{Estimation of the stray field of the tip}

Despite each MNP has its own switching field (depending on their size, shape, orientation of the easy axis and dipolar interactions), from SQUID magnetometer measurements of $\mathrm{K}_{0.22} \mathrm{Ni}\left[\mathrm{Cr}(\mathrm{CN})_{6}\right]_{0.74} \mathrm{MNPs}$ powder dispersed in a polymeric matrix, an averaged MNP coercive field of \pm 170 Oe per MNP can be estimated at $2 \mathrm{~K}$ (from reference 2). Taking this average value, we would need an external magnetic field larger than +170 Oe to start switching the magnetization of a MNP that is magnetized in the opposite direction. Analysing the magnetic images shown in figure 2 in the manuscript, we observe that the switching occurs in a range of $B_{\text {ext }}$ between -400 Oe and -200 Oe, which means that the straight field 
of the tip is compensating this negative values plus 170 Oe. Therefore, we could roughly estimate the stray field of the tip to be between 570 Oe and 370 Oe.

\section{S.I. 2 LT-MFM measurements of the different vortex state configurations in single MNPs}

a

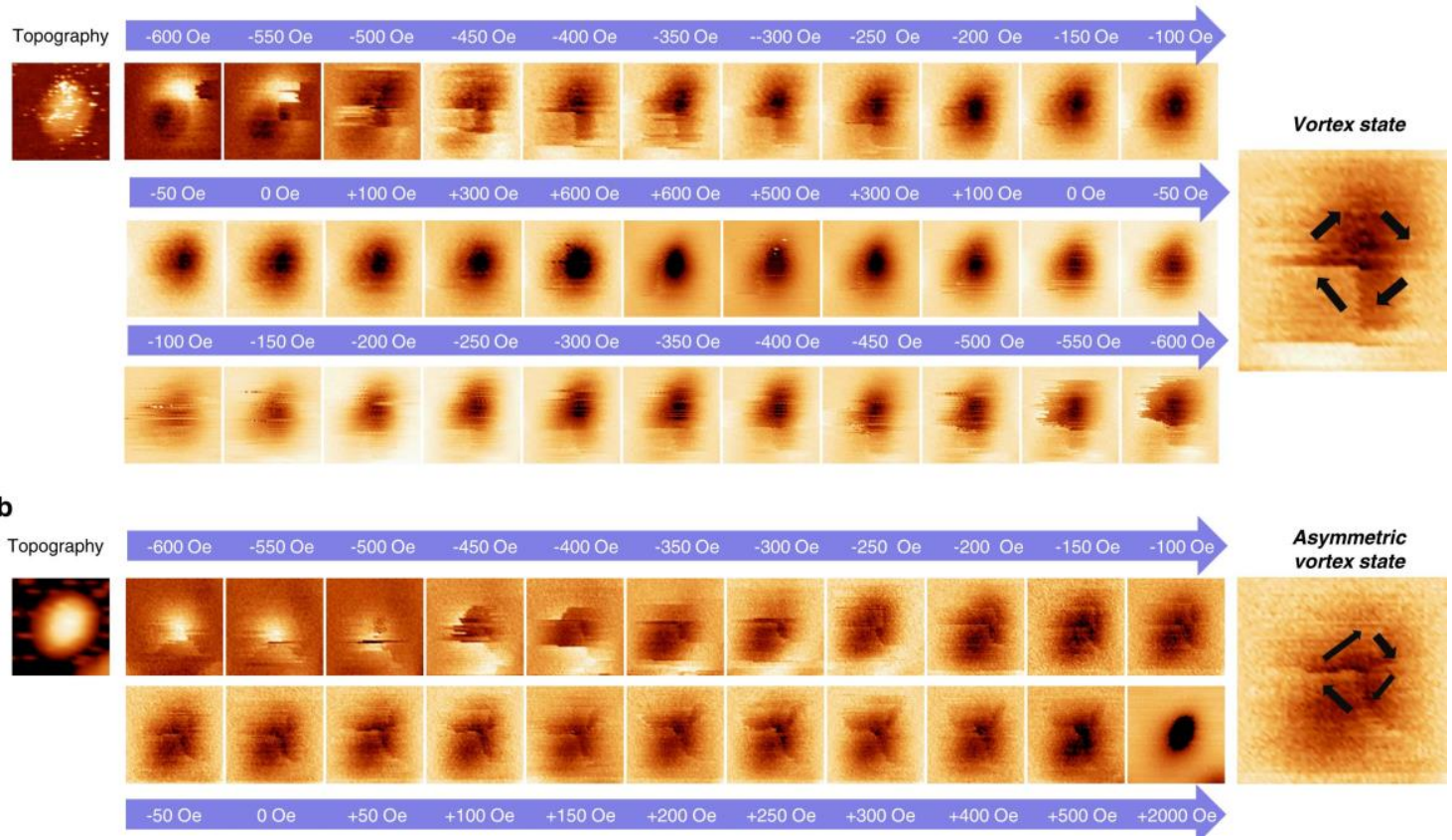

Figure S2. (a) Left: LT-MFM Images of the topography and magnetization reversal of a single MNP of $23 \mathrm{~nm}$ height. The gradual change in the magnetic contrast shows the in-plane magnetic circulation of the vortex structure. Right: Enlarged magnetic image (at -300 Oe) showing the vortex state. (b) Left: LT-MFM Images of the topography and magnetization reversal of a single MNP of $28 \mathrm{~nm}$ height. The magnetic contrast reveals the formation of an asymmetric vortex state. Right: enlarged magnetic image (at +50 Oe) showing the asymmetric vortex state. (Size of all images: $112 \mathrm{~nm} \times 112$ $\mathrm{nm})$. 